\title{
Applications of the loop-tree duality
}

\author{
Germán Rodrigo*, Félix Driencourt-Mangin, Germán F. R. Sborlini \\ Instituto de Física Corpuscular, Universitat de València - Consejo Superior de Investigaciones \\ Científicas, Parc Científic, E-46980 Paterna, Valencia, Spain \\ E-mail: german.rodrigoecsic.es, felix.dmeific.uv.es, \\ german.sborlinilific.uv.es
}

\section{Roger José Hernández-Pinto}

Facultad de Ciencias Físico-Matemáticas, Universidad Autónoma de Sinaloa, Ciudad

Universitaria, CP 80000, Culiacán, Sinaloa, México

E-mail: roger@uas.edu.mx

\begin{abstract}
We describe a new method to perform NLO calculations, combining real and virtual amplitudes at the integrand level, with a fully local compensation between them in the IR, and between the virtual integrand and properly defined counter-terms in the UV, in such a way that physical observables can be computed in 4 dimensions. One of the advantages of the method is that all the scattering amplitudes are integrated simultaneously, without the need for tensor reduction, or projection onto sets of master integrals. As such, it could offer great progress in the automation of NLO calculations, where the actual bottle-necks are the complexity of the analytical calculations for multi-leg processes, and the numerical stability of the result.
\end{abstract}

Loops and Legs in Quantum Field Theory

24-29 April 2016

Leipzig, Germany

\footnotetext{
* Speaker.
} 


\section{The loop-tree duality}

The standard approach to perform perturbative calculation in QCD relies in the application of the subtraction formalism. There are several variants of the subtraction method at NLO and beyond $[1,2,3,4,5,6,7,8,9,10,11]$, which involve treating separately real and virtual contributions. Besides the computational difficulty related to the fact that the final-state phase-space of the different contributions involves different number of particles, building these counter-terms represents a challenge and introduces a potential bottleneck to efficiently carry out the infrared (IR) subtraction for multi-leg multi-loop processes.

With the aim of eluding the introduction of IR counter-terms, we have developed an alternative idea based on the application of the loop-tree duality (LTD) $[12,13,14,15,16,17,18,19,20,21$, $22]^{1}$. The LTD theorem establishes that loop scattering amplitudes, at one-loop and beyond, can be expressed as a sum of phase-space integrals (i.e. the so-called dual integrals) with additional physical particles. Dual integrals and real-radiation contributions exhibit a similar structure, and can be combined at integrand level. For example, the dual representation of the scalar one-loop integral over $N$ Feynman propagators, $G_{F}\left(q_{i}\right)$, is the sum of $N$ dual integrals [12, 13]:

$$
L^{(1)}\left(\left\{p_{i}\right\}\right)=\int_{\ell} \prod_{i \in \alpha_{1}} G_{F}\left(q_{i}\right)=-\sum_{i \in \alpha_{1}} \int_{\ell} \tilde{\delta}\left(q_{i}\right) \prod_{j \in \alpha_{1}, j \neq i} G_{D}\left(q_{i} ; q_{j}\right), \quad \alpha_{1}=\{1, \cdots, N\},
$$

where

$$
G_{D}\left(q_{i} ; q_{j}\right)=\frac{1}{q_{j}^{2}-m_{j}^{2}-\imath 0 \eta k_{j i}}
$$

are the so-called dual propagators, with $\eta$ a future-like vector, $\eta^{2} \geq 0$, with positive definite energy $\eta_{0}>0$. The delta function $\tilde{\delta}\left(q_{i}\right) \equiv 2 \pi_{\imath} \theta\left(q_{i, 0}\right) \delta\left(q_{i}^{2}-m_{i}^{2}\right)$ sets the internal lines on-shell by selecting the pole of the Feynman propagators with positive energy $q_{i, 0}$ and negative imaginary part. The sign of the $\imath 0$ prescription depends now on $k_{j i}=q_{j}-q_{i}$, which indeed at one-loop is a function of the external momenta $p_{i}$ only. Having different $\iota 0$ prescriptions for each cut, far from introducing an extra difficulty, is a necessary condition for the consistency of the method. Actually, it is needed to demonstrate the main property of LTD, namely the partial cancellation of singularities among different dual integrands and the causal interpretation of the remaining ones [16].

Regular loop integrals become singular at the on-shell hyperboloids (light-cones for massless propagators) of the Feynman propagators, $G_{F}^{-1}\left(q_{i}\right)=0$. The first advantage of LTD is that integration over the loop momentum is directly set on top of the forward on-shell hyperboloids (positive energy modes). Singularities in the loop three-momentum space appear only in the intersection with other forward or backward (negative energy modes) on-shell hyperboloids. However, singularities in the intersection of forward on-shell hyperboloids cancel among different dual contributions; the change of sign of the modified $\imath 0$ prescription is crucial to enable this behaviour. Only singularities in the intersection of forward with backward on-shell hyperboloids (FB) remain, and even more important, they are constrained to a compact region of the loop-three momentum space and are easily reinterpreted in terms of causality. From a physical point of view, FB singularities take place when the on-shell virtual particle interacts with another on-shell virtual particle

\footnotetext{
${ }^{1}$ See also Ref. [23].
} 
after the emission of outgoing on-shell radiation. The direction of the internal momentum flow establishes a natural causal ordering, and this interpretation is consistent with the Cutkosky rule.

The compactness of the singular region is crucial to advance one steep further in the implementation of the method. Threshold singularities are integrable, and can be integrated numerically by contour deformation $[16,17,18]$. The treatment of IR singularities is discussed in the next Section.

\section{Four dimensional unsubtraction}

The summation over degenerate soft and collinear states from virtual and real corrections is implemented in LTD by a suitable mapping of momenta between the virtual and real kinematics. This is possible because the IR singularities are restricted to a compact region of the loop threemomentum, and makes unnecessary the introduction of IR subtractions for soft and final-state collinear singularities. As usual, the NLO cross-section is constructed from the one-loop virtual correction with $m$ partons in the final state and the exclusive real cross-section with $m+1$ partons in the final state

$$
\sigma^{\mathrm{NLO}}=\int_{m} d \sigma_{\mathrm{V}}^{(1, \mathrm{R})}+\int_{m+1} d \sigma_{\mathrm{R}}^{(1)}
$$

where the virtual contribution is obtained from its dual representation

$$
d \sigma_{\mathrm{V}}^{(1, \mathrm{R})}=\sum_{i=1}^{N} \int_{\ell} 2 \operatorname{Re}\left\langle\mathscr{M}_{N}^{(0)} \mid \mathscr{M}_{N}^{(1, \mathrm{R})}\left(\tilde{\boldsymbol{\delta}}\left(q_{i}\right)\right)\right\rangle \mathscr{O}_{N}\left(\left\{p_{i}\right\}\right)
$$

In Eq. (2.2), $\mathscr{M}_{N}^{(0)}$ is the $N$-leg scattering amplitude at LO, and $\mathscr{M}_{N}^{(1, \mathrm{R})}$ is the renormalised oneloop scattering amplitude, which also contains the self-energy corrections of the external legs, even if they are massless and then ignored in the usual calculations because their integrated form vanishes. The delta function $\tilde{\delta}\left(q_{i}\right)$ symbolises the dual contribution with the internal momentum $q_{i}$ set on-shell. The integral is weighted with the function $\mathscr{O}_{N}$ that defines a given observable, for example the jet cross-section in the $k_{T}$-algorithm. The renormalised amplitude includes appropriate counter-terms that subtract the UV singularities locally, as discussed in Ref. [22], including UV singularities of degree higher than logarithmic that integrate to zero. In Eq. (2.2), we have also assumed a definite ordering of the external particles that leads to a definite set of internal momenta.

The real cross-section is given by

$$
\int_{m+1} d \sigma_{\mathrm{R}}^{(1)}=\sum_{i=1}^{N} \int_{m+1}\left|\mathscr{M}_{N+1}^{(0)}\left(q_{i}, p_{i}\right)\right|^{2} \mathscr{R}_{i}\left(q_{i}, p_{i}\right) \mathscr{O}_{N+1}\left(\left\{p_{j}^{\prime}\right\}\right),
$$

where the external momenta $p_{j}^{\prime}$, the phase-space and the tree-level scattering amplitude $\mathscr{M}_{N+1}^{(0)}$ are rewritten in terms of the loop three-momentum (equivalently, the internal on-shell loop momenta) and the external momenta $p_{i}$ of the Born process.

Analogously to the dipole method [3, 4], we single out two partons for each contribution in order to construct the momentum mapping between the $m$ and $m+1$ kinematics. The first parton is the emitter and the second parton is the spectator. Then, the loop three-momentum and the four-momenta of the emitter and the spectator are used to reconstruct the kinematics of the 

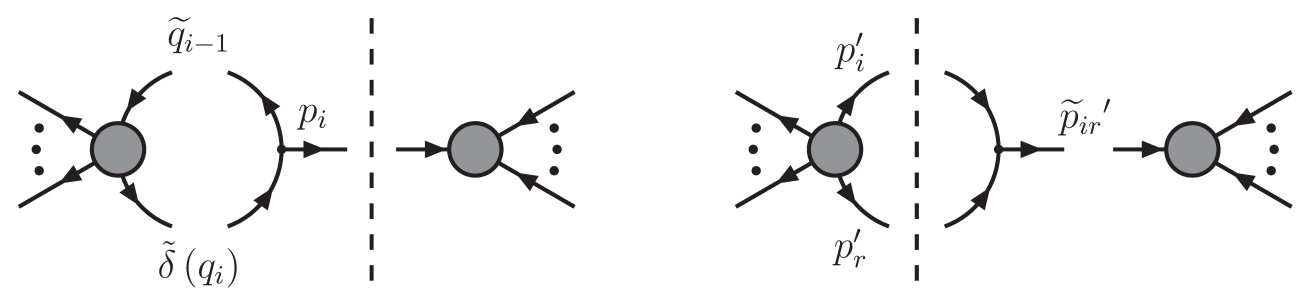

Figure 1: Interference of the Born process with the one-loop scattering amplitude with internal momentum $q_{i}$ on-shell, $\mathscr{M}_{N}^{(1)}\left(\tilde{\delta}\left(q_{i}\right)\right) \otimes \mathscr{M}_{N}^{(0) \dagger}$ (left), and interference of real processes with parton splitting $p_{i r}^{\prime} \rightarrow p_{i}^{\prime}+$ $p_{r}^{\prime}: \mathscr{M}_{N+1}^{(0)} \otimes \mathscr{M}_{N+1}^{(0) \dagger}\left(p_{i r}^{\prime}\right)$ (right). The dashed line represents momentum conservation. In the soft/collinear limits the momenta $q_{i-1}=q_{i}-p_{i}$ and $p_{i r}^{\prime}$ become on-shell and the scattering amplitudes factorise.

corresponding real emission cross-section in the region of the real phase-space where the twin of the emitter decays to two partons in a soft or collinear configuration. Explicitly, if the momentum of the final-state emitter is $p_{i}$, the internal on-shell momentum prior to the emitter is $q_{i}$, and $p_{j}$ is the momentum of the final-state spectator, then, the momentum mapping is given by

$$
\begin{array}{rlrl}
p_{r}^{\prime \mu} & =q_{i}^{\mu}, & \\
p_{i}^{\prime \mu} & =p_{i}^{\mu}-q_{i}^{\mu}+\alpha_{i} p_{j}^{\mu}, & & \alpha_{i}=\frac{\left(q_{i}-p_{i}\right)^{2}}{2 p_{j} \cdot\left(q_{i}-p_{i}\right)}, \\
p_{j}^{\prime \mu} & =\left(1-\alpha_{i}\right) p_{j}^{\mu}, & & \\
p_{k}^{\prime \mu} & =p_{k}^{\mu}, & k \neq i, j .
\end{array}
$$

The incoming initial-state momenta, $p_{a}$ and $p_{b}$, are left unchanged, and $p_{r}^{\prime}$ is the momentum of the extra radiated particle. Momentum conservation is automatically fulfilled by Eq. (2.4) because $p_{i}+$ $p_{j}+\sum_{k \neq i, j} p_{k}=p_{i}^{\prime}+p_{r}^{\prime}+p_{j}^{\prime}+\sum_{k \neq i, j} p_{k}^{\prime}$, and all the primed final-state momenta are massless and on-shell if the virtual unprimed momenta are also massless. The momentum mapping in Eq. (2.4) is motivated by the general factorisation properties in QCD [16, 24], and it is graphically explained in Fig. 1.

The emitter $p_{i}$ has the same flavour as $p_{i r}^{\prime}$, the twin emitter or parent parton (called emitter in the dipole formalism) of the real splitting configuration that is mapped. The spectator $p_{j}$ is used to balance momentum conservation, and has the same flavour in the virtual and real contributions. Similar mappings, involving also initial-state momenta, have been presented recently in Ref. [23]. As for the dipoles, there are alternatives to treat the recoiling momentum, but the option with a single spectator is the simplest one.

The momentum mapping in Eq. (2.4) is suitable in the region of the loop-momentum space where $q_{i}$ is soft or collinear with $p_{i}$, and therefore in the region of the real phase-space where $p_{i}^{\prime}$ and $p_{r}^{\prime}$ are produced collinear or one of them is soft. In Eq. (2.2), we have introduced a complete partition of the real phase-space

$$
\sum \mathscr{R}_{i}\left(q_{i}, p_{i}\right)=\sum \prod_{j k \neq i r} \theta\left(y_{j k}^{\prime}-y_{i r}^{\prime}\right)=1
$$

which is equivalent to split the phase-space as a function of the minimal dimensionless two-body invariant $y_{i r}^{\prime}=s_{i r}^{\prime} / s$. Since the real and virtual kinematics are related, the real phase-space par- 
tition defines equivalent regions in the loop three-momentum space. Notice, however, that we have not imposed these constraints in the definition of the virtual cross-section in Eq. (2.2). The actual implementation of the NLO cross-section in a Monte Carlo event generator is a single unconstrained integral in the loop three-momentum, and the phase-space with $m$ final-state particles. By virtue of the momentum mapping, real corrections are contributing in the region of the loop three-momentum where they map the corresponding soft and collinear divergences. This region is compact, and it is of the size of the representative hard scale of the scattering process. At large loop three-momentum, only the virtual corrections contribute, and their UV singularities are subtracted locally by suitable counter-terms. The IR singularities are unsubtracted because their cancellation is achieved at the integrand level by combining simultaneously virtual and real corrections. The momentum mapping respects the soft and collinear limits, therefore the IR cancellation is fulfilled for infrared safe observables and not only for the total cross-section. The full calculation can be implemented in four-dimensions, more precisely with the Dimensional Regularization (DREG) parameter $\varepsilon=0$.

At NNLO, the total cross-section consists of three contributions

$$
\sigma^{\mathrm{NNLO}}=\int_{m} d \sigma_{\mathrm{VV}}^{(2)}+\int_{m+1} d \sigma_{\mathrm{VR}}^{(2)}+\int_{m+2} d \sigma_{\mathrm{RR}}^{(2)},
$$

where the double virtual cross-section $d \sigma_{\mathrm{VV}}^{(2)}$ receives contributions from the interference of the two-loop with the Born scattering amplitudes, and the square of the one-loop scattering amplitude with $m$ final-state particles, the virtual-real cross-section $d \sigma_{\mathrm{VR}}^{(2)}$ includes the contributions from the interference of one-loop and tree-level scattering amplitudes with one extra external particle, and the double real cross-section $d \sigma_{\mathrm{RR}}^{(2)}$ are tree-level contributions with emission of two extra particles. The LTD representation of the two-loop scattering amplitude is obtained by setting two internal lines on-shell [13]. It leads to the two-loop dual components $\left\langle\mathscr{M}_{N}^{(0)} \mid \mathscr{M}_{N}^{(2)}\left(\tilde{\delta}\left(q_{i}\right), \tilde{\delta}\left(q_{j}\right)\right)\right\rangle$, while the two-loop momenta of the squared one-loop amplitude are independent and generate dual contributions of the type $\left\langle\mathscr{M}_{N}^{(1)}\left(\tilde{\delta}\left(q_{i}\right)\right) \mid \mathscr{M}_{N}^{(1)}\left(\tilde{\delta}\left(q_{j}\right)\right)\right\rangle$. In both cases, there are two independent loop three-momenta and $m$ final-state momenta, from where we can reconstruct the kinematics of the one-loop corrections entering $d \sigma_{\mathrm{VR}}^{(2)}$, and the tree-level corrections in $d \sigma_{\mathrm{RR}}^{(2)}$.

\section{NLO corrections to $\gamma^{*} \rightarrow q \bar{q}(g)$ in four dimensions}

We present in this section the four-dimensional representation of the total cross-section for the physical process $\gamma^{*} \rightarrow q \bar{q}(g)$ at NLO. The loop internal momenta are $q_{1}=\ell+p_{1}, q_{2}=\ell+p_{12}$ and $q_{3}=\ell$, which are parametrised as

$$
q_{i}^{\mu}=\frac{\sqrt{s_{12}}}{2} \xi_{1,0}\left(1,2 \sqrt{v_{i}\left(1-v_{i}\right)} \mathbf{e}_{i, \perp}, 1-2 v_{i}\right)
$$

when they are set on-shell, $q_{i}^{2}=0$. The direct combination of the virtual and real corrections leads to the dual cross-section contributions

$$
\tilde{\sigma}_{1}^{(1)}=\sigma^{(0)} \frac{\alpha_{\mathrm{S}}}{4 \pi} C_{F} \int_{0}^{1} d \xi_{1,0} \int_{0}^{1 / 2} d v_{1} 4 \mathscr{R}_{1}\left(\xi_{1,0}, v_{1}\right)\left[2\left(\xi_{1,0}-\left(1-v_{1}\right)^{-1}\right)-\frac{\xi_{1,0}\left(1-\xi_{1,0}\right)}{\left(1-\left(1-v_{1}\right) \xi_{1,0}\right)^{2}}\right],
$$



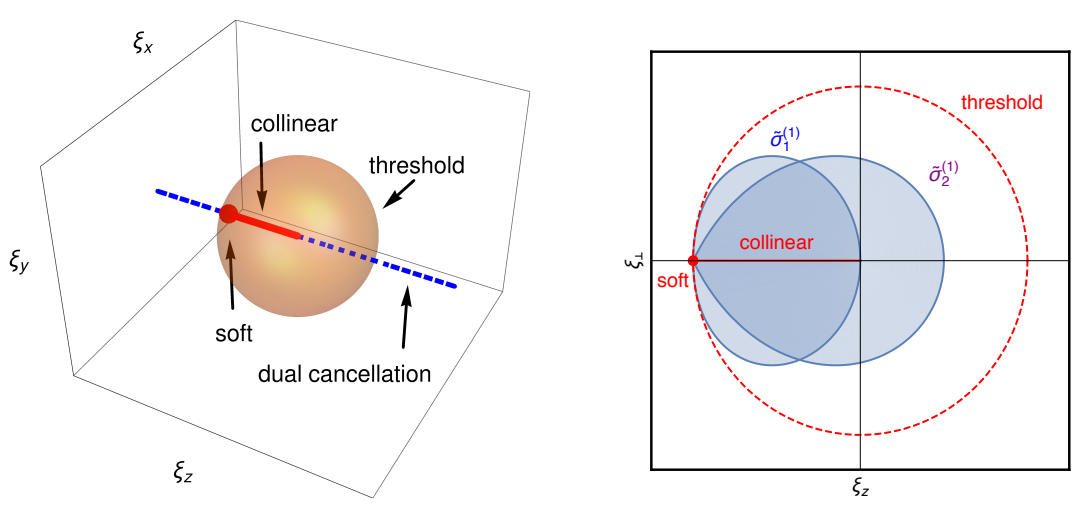

Figure 2: Singular structure of the three-point function in the loop coordinates $\ell^{\mu}=\sqrt{s_{12}} / 2 \xi^{\mu}$ (left plot); regions in the loop three-momentum space (right plot) where virtual and real kinematics are mapped, with $\xi_{\perp}=\sqrt{\xi_{x}^{2}+\xi_{y}^{2}}$.

$$
\begin{aligned}
\widetilde{\sigma}_{2}^{(1)}= & \sigma^{(0)} \frac{\alpha_{\mathrm{S}}}{4 \pi} C_{F} \int_{0}^{1} d \xi_{2,0} \int_{0}^{1} d v_{2} 2 \mathscr{R}_{2}\left(\xi_{2,0}, v_{2}\right)\left(1-v_{2}\right)^{-1}\left[\frac{2 v_{2} \xi_{2,0}\left(\xi_{2,0}\left(1-v_{2}\right)-1\right)}{1-\xi_{2,0}}\right. \\
& \left.-1+v_{2} \xi_{2,0}+\frac{1}{1-v_{2} \xi_{2,0}}\left(\frac{\left(1-\xi_{2,0}\right)^{2}}{\left(1-v_{2} \xi_{2,0}\right)^{2}}+\xi_{2,0}^{2}\right)\right],
\end{aligned}
$$

where the integration regions are defined through

$$
\begin{aligned}
& \mathscr{R}_{1}\left(\xi_{1,0}, v_{1}\right)=\theta\left(1-2 v_{1}\right) \theta\left(\frac{1-2 v_{1}}{1-v_{1}}-\xi_{1,0}\right), \\
& \mathscr{R}_{2}\left(\xi_{2,0}, v_{2}\right)=\theta\left(\frac{1}{1+\sqrt{1-v_{2}}}-\xi_{2,0}\right) .
\end{aligned}
$$

A graphical representation of these regions is shown in Fig. 2 (right). Outside these regions only virtual corrections contribute, and the sum of all the dual integrals is finite after the inclusion of suitable UV counter-term. The dual remnant is

$$
\begin{aligned}
\bar{\sigma}_{\mathrm{V}}^{(1)} & =\sigma^{(0)} \frac{\alpha_{\mathrm{S}}}{4 \pi} C_{F} \int_{0}^{\infty} d \xi \int_{0}^{1} d v\left\{-2\left(1-\mathscr{R}_{1}(\xi, v)\right) v^{-1}(1-v)^{-1} \frac{\xi^{2}(1-2 v)^{2}+1}{\sqrt{(1+\xi)^{2}-4 v \xi}}\right. \\
& +2\left(1-\mathscr{R}_{2}(\xi, v)\right)(1-v)^{-1}\left[2 v \xi(\xi(1-v)-1)\left(\frac{1}{1-\xi+\imath 0}+\imath \pi \delta(1-\xi)\right)-1+v \xi\right] \\
& +2 v^{-1}\left(\frac{\xi(1-v)(\xi(1-2 v)-1)}{1+\xi}+1\right)-\frac{(1-2 v) \xi^{3}\left(12-7 m_{\mathrm{UV}}^{2}-4 \xi^{2}\right)}{\left(\xi^{2}+m_{\mathrm{UV}}^{2}\right)^{5 / 2}} \\
& \left.-\frac{2 \xi^{2}\left(m_{\mathrm{UV}}^{2}+4 \xi^{2}(1-6 v(1-v))\right)}{\left(\xi^{2}+m_{\mathrm{UV}}^{2}\right)^{5 / 2}}\right\} .
\end{aligned}
$$

In the previous expression, we have identified all the integration variables, $\xi=\xi_{2,0}=\xi_{3,0}=\xi_{\mathrm{UV}}$ and $v=v_{2}=v_{3}=v_{\mathrm{UV}}$, while $\left(\xi_{1,0}, v_{1}\right)$ are expressed in terms of $\left(\xi_{2,0}, v_{2}\right)$ with the appropriate change of variables [22]. The last two terms in Eq. (3.4) that depend on $m_{\mathrm{UV}}^{2}$ are the UV 
counter-terms of the vertex and the self-energies. Putting together the three dual contributions from Eq. (3.2) and Eq. (3.4), we obtain

$$
\sigma=\sigma^{(0)}\left(1+3 C_{F} \frac{\alpha_{\mathrm{S}}}{4 \pi}+\mathscr{O}\left(\alpha_{\mathrm{S}}^{2}\right)\right)
$$

which agrees with the well-known result available in the literature. Notice that it was unnecessary to introduce any tensor reduction; Gram determinants are naturally avoided in LTD, and therefore the spurious singularities that the tensor reduction introduces leading to numerical instabilities in the integration over the phase-space.

\section{Conclusions and outlook}

We have discussed the implementation of a novel algorithm to compute higher-order corrections to physical observables. This method is based on the LTD theorem, which states that virtual contributions can be expressed as the sum over single-cut (at one-loop) or dual integrals, whose structure closely resembles real-emission amplitudes. Moreover, in LTD all the threshold and IR divergences are generated in a compact region of the loop three-momentum space. The summation over degenerate soft and collinear configurations is achieved by defining suitable momentum mapping relating the virtual and real kinematics. These mappings correlate exactly the kinematical configurations in the integration regions where the IR singularities are originated. In the high-energy region unintegrated UV counter-terms cancel the UV singular behaviour, their LTD representations have been also presented in detail, and admit a natural physical interpretation. The algorithm is called unsubtracted because the momentum mappings make unnecessary the introduction of IR subtractions.

These facts can be exploited to perform an integrand-level combination of real and virtual terms, which leads to a fully local cancellation of singularities and allows to implement NLO calculations without making use of DREG. It constitutes a new paradigm in perturbative calculations because it takes advantage of combining directly real and virtual corrections in an integrable fourdimensional representation, while providing an easy physical interpretation of the singularities of the scattering amplitudes and unveil their hidden nature.

The implementation of the method has been illustrated with the simplest physical process $\gamma^{*} \rightarrow q \bar{q}(g)$ at NLO in QCD, and explicit expressions in four dimensions have been presented. We have also succinctly sketched the generalisation to NNLO, and are currently working in the extension of the method to deal with massive particles [25]. The numerical treatment of threshold singularities by contour deformation has also been investigated recently in Refs. [16, 17, 18]

\section{Acknowledgements}

This work has been supported by CONICET Argentina, by the Spanish Government and ERDF funds from the European Commission (Grants No. FPA2014-53631-C2-1-P and SEV-2014-0398) and by Generalitat Valenciana under Grant No. PROMETEOII/2013/007. FDM acknowledges suport from Generalitat Valenciana (GRISOLIA/2015/035). The work of RJHP is partially supported by CONACyT, México. 


\section{References}

[1] Z. Kunszt and D. E. Soper, Phys. Rev. D 46 (1992) 192.

[2] S. Frixione, Z. Kunszt and A. Signer, Nucl. Phys. B 467 (1996) 399 [hep-ph/9512328].

[3] S. Catani and M. H. Seymour, Phys. Lett. B 378 (1996) 287 [hep-ph/9602277].

[4] S. Catani and M. H. Seymour, Nucl. Phys. B 485 (1997) 291 [Nucl. Phys. B 510 (1998) 503] [hep-ph/9605323].

[5] A. Gehrmann-De Ridder, T. Gehrmann and E. W. N. Glover, JHEP 0509 (2005) 056 [hep-ph/0505111].

[6] S. Catani and M. Grazzini, Phys. Rev. Lett. 98 (2007) 222002 [hep-ph/0703012].

[7] M. Czakon, Phys. Lett. B 693 (2010) 259 [arXiv:1005.0274 [hep-ph]].

[8] P. Bolzoni, G. Somogyi and Z. Trocsanyi, JHEP 1101 (2011) 059 [arXiv:1011.1909 [hep-ph]].

[9] V. Del Duca, C. Duhr, G. Somogyi, F. Tramontano and Z. Trócsányi, JHEP 1504 (2015) 036 [arXiv:1501.07226 [hep-ph]].

[10] R. Boughezal, C. Focke, X. Liu and F. Petriello, Phys. Rev. Lett. 115 (2015) 062002. [arXiv:1504.02131 [hep-ph]].

[11] J. Gaunt, M. Stahlhofen, F. J. Tackmann and J. R. Walsh, JHEP 1509 (2015) 058. [arXiv:1505.04794 [hep-ph]].

[12] S. Catani, T. Gleisberg, F. Krauss, G. Rodrigo and J. C. Winter, JHEP 0809 (2008) 065 [arXiv:0804.3170 [hep-ph]].

[13] I. Bierenbaum, S. Catani, P. Draggiotis and G. Rodrigo, JHEP 1010 (2010) 073 [arXiv:1007.0194 [hep-ph]].

[14] I. Bierenbaum, S. Buchta, P. Draggiotis, I. Malamos and G. Rodrigo, JHEP 1303 (2013) 025 [arXiv:1211.5048 [hep-ph]].

[15] I. Bierenbaum, P. Draggiotis, S. Buchta, G. Chachamis, I. Malamos and G. Rodrigo, Acta Phys. Polon. B 44 (2013) 2207.

[16] S. Buchta, G. Chachamis, P. Draggiotis, I. Malamos and G. Rodrigo, JHEP 1411 (2014) 014 [arXiv:1405.7850 [hep-ph]].

[17] S. Buchta, PhD thesis, Universitat de València, 2015, arXiv:1509.07167 [hep-ph].

[18] S. Buchta, G. Chachamis, P. Draggiotis and G. Rodrigo, arXiv:1510.00187 [hep-ph].

[19] R. J. Hernández-Pinto, G. F. R. Sborlini and G. Rodrigo, JHEP 1602 (2016) 044 [arXiv:1506.04617 [hep-ph]].

[20] G. F. R. Sborlini, R. Hernández-Pinto and G. Rodrigo, PoS EPS-HEP 2015 (2015) 479 [arXiv:1510.01079 [hep-ph]].

[21] G. F. R. Sborlini, PoS RADCOR 2015 (2015) 082 [arXiv:1601.04634 [hep-ph]].

[22] G. F. R. Sborlini, F. Driencourt-Mangin, R. Hernandez-Pinto and G. Rodrigo, JHEP 1608 (2016) 160 [arXiv:1604.06699 [hep-ph]].

[23] S. Seth and S. Weinzierl, Phys. Rev. D 93 (2016) 114031 [arXiv:1605.06646 [hep-ph]].

[24] S. Catani, D. de Florian and G. Rodrigo, JHEP 1207 (2012) 026 [arXiv:1112.4405 [hep-ph]].

[25] G. F. R. Sborlini, F. Driencourt-Mangin and G. Rodrigo, arXiv:1608.01584 [hep-ph]. 